\title{
DISTRIBUTIONAL AND $L^{q}$ NORM INEQUALITIES FOR POLYNOMIALS OVER CONVEX BODIES IN $\mathbb{R}^{n}$
}

\author{
Anthony CARBerY* And James Wright**
}

This paper is dedicated to the memory of Donna L. Wright.

\section{Introduction}

Let $\mathcal{P}_{d, n}$ be the vector space of all polynomials of degree at most $d$ in $\mathbb{R}^{n}$. Let $K$ be a convex body of volume 1 in $\mathbb{R}^{n}$ and let $1 \leq q \leq \infty$. Since $\mathcal{P}_{d, n}$ is finite dimensional, the norms $\left(\int_{K}|p|^{q}\right)^{\frac{1}{q}}$ are all equivalent to each other. Recently there has been considerable interest in the behaviour of the constants in these equivalences as $q$ varies when we consider arbitrary unit-volume convex bodies $K$. See for example the work of Brudnyi and Ganzburg [BG], Gromov and Milman [GM], Bourgain [Bour], Bobkov [Bobk] and Nazarov, Sodin and Volberg [NSV].

In this paper, we wish to complete the analysis of the constants in these equivalences as well as to extend these results to the vector-valued setting. For a (real or complex) Banach space $X$ with norm $\|\cdot\|$ and a polynomial $p: \mathbb{R}^{n} \rightarrow X$ of degree at most $d$, we define the functional $p^{\#}(x)=\|p(x)\|^{\frac{1}{d}}$. For a convex body $K$ in $\mathbb{R}^{n}$ of volume 1 , we consider the usual $L^{q}$ norms of $p^{\#}$ over $K$; that is, $\left\|p^{\#}\right\|_{q}=\left(\int_{K} p^{\#}(x)^{q} d x\right)^{\frac{1}{q}}=\left(\int_{K}\|p(x)\|^{\frac{q}{d}} d x\right)^{\frac{1}{q}}$. When $q=0$, we set $\left\|p^{\#}\right\|_{0}=\exp \int_{K} \log p^{\#}(x) d x$ and $\left\|p^{\#}\right\|_{\infty}$ is the usual $L^{\infty}$ norm of $p^{\#}$.

Let $0 \leq r \leq q \leq \infty$. Hölder's inequality gives a trivial inequality for the $L^{q}$ norms with (best possible) constant 1 and for the reverse inequality we have:

Theorem 1. Let $p: \mathbb{R}^{n} \rightarrow X$ be a polynomial of degree at most $d$, let $K$ be a convex body in $\mathbb{R}^{n}$ of volume 1 and let $0 \leq r \leq q \leq \infty$. Then there exists an absolute constant $C$ independent of $p, d, K, n, q, r$ and $X$ such that

$$
\left\|p^{\#}\right\|_{q} \leq C \frac{[n B(n, q+1)]^{\frac{1}{q}}}{[n B(n, r+1)]^{\frac{1}{r}}}\left\|p^{\#}\right\|_{r}
$$

where $B$ denotes the classical Beta function.

Received November 7, 2000.

* Supported by a Leverhulme Fellowship.

** Supported in part by an ARC grant. 
Recall that $n B(n, q+1)=-\int_{0}^{1} u^{q} d(1-u)^{n}$; in the limiting cases $q=0$ and $q=\infty$, the quantity $[n B(n, q+1)]^{\frac{1}{q}}$ is to be understood as $1 / n$ and 1 respectively. In particular we note that the estimate in Theorem 1 is independent of the norm $\|\cdot\|$ from $X$.

By standard estimates for the Beta function we obtain:

Corollary. Let $p: \mathbb{R}^{n} \rightarrow X$ be a polynomial of degree at most $d$, let $K$ be a convex body in $\mathbb{R}^{n}$ of volume 1 and let $0 \leq r \leq q \leq \infty$. Then there exists an absolute constant $C$ independent of $p, d, K, n, q, r$ and $X$ such that

(a) if $n \leq r \leq q$ then

$$
\left\|p^{\#}\right\|_{q} \leq C\left\|p^{\#}\right\|_{r}
$$

(b) if $r \leq n \leq q$ then

$$
\left\|p^{\#}\right\|_{q} \leq C \frac{n}{\max (r, 1)}\left\|p^{\#}\right\|_{r}
$$

(c) if $r \leq q \leq n$ then

$$
\left\|p^{\#}\right\|_{q} \leq C \frac{\max (q, 1)}{\max (r, 1)}\left\|p^{\#}\right\|_{r}
$$

Up to the numerical constant $C$, the constant on the right hand side of Theorem 1 is optimal if one seeks an inequality valid for arbitrary convex bodies $K$. One simply takes $p(x)=x_{1}^{d}$ and $K=\left\{\left(x_{1}, x^{\prime}\right) \in \mathbb{R}^{n}: 0 \leq x_{1} \leq 1,\left|x^{\prime}\right| \leq 1-x_{1}\right\}$. The scalar-valued case $q=\infty, r \leq 1$ (in which case the constant on the right hand side is essentially $n$ ) is due to Brudnyi and Ganzburg [BG]. For dimensionless bounds, the scalar-valued cases $r=0, q \geq 0$ and $r=d, q \geq 2 d$ are due to Bobkov [Bobk] (in these cases the dimensionless bound on the right hand side is essentially $q$ ). One can then extrapolate these bounds to get sharp dimension free Khinchine-Kahane type inequalities in the exponential class. This refined earlier work of Bourgain [Bour] which in turn extended a result of Gromov and Milman $[\mathrm{GM}]$ to the general degree $d$ case from the linear case $d=1$. Nazarov, Sodin and Volberg [NSV] have also obtained Bobkov's dimensionless bound in the case $r=0$ and $q \geq 0$ (by different methods), as well as other interesting results. Our Theorem 1 may be viewed as a completion of all these results, giving the precise behaviour in all the parameters, $d, n, q$ and $r$.

The case $r \leq 1$ and general $q$ has a stronger formulation in terms of distributional inequalities for vector-valued polynomials over convex bodies in $\mathbb{R}^{n}$ (which may be of independent interest for certain problems in real and harmonic analysis). In fact, we have:

Theorem 2. Let $p: \mathbb{R}^{n} \rightarrow X$ be a polynomial of degree at most $d$, and let $K$ be a convex body in $\mathbb{R}^{n}$ of volume 1 . Let $0 \leq q \leq \infty$. Then there exists an absolute constant $C$ independent of $p, d, K, n, q$ and $X$ so that for any $\alpha>0$,

$$
\left\|p^{\#}\right\|_{q} \alpha^{-1}\left|\left\{x \in K: p^{\#}(x) \leq \alpha\right\}\right| \leq C n(n B(n, q+1))^{\frac{1}{q}} .
$$


In particular, we have:

Corollary. Let $p: \mathbb{R}^{n} \rightarrow X$ be a polynomial of degree at most $d$, let $K$ be a convex body in $\mathbb{R}^{n}$ of volume 1 and let $0 \leq q \leq \infty$. Then there exists an absolute constant $C$ independent of $p, d, K, n, q$ and $X$ so that for any $\alpha>0$,

(a) if $n \leq q$ then

$$
\left\|p^{\#}\right\|_{q} \alpha^{-1}\left|\left\{x \in K: p^{\#}(x) \leq \alpha\right\}\right| \leq C n ;
$$

(b) if $q \leq n$ then

$$
\left\|p^{\#}\right\|_{q} \alpha^{-1}\left|\left\{x \in K: p^{\#}(x) \leq \alpha\right\}\right| \leq C \max (q, 1) .
$$

As before, up to the constant $C$, the inequalities are sharp (to see this we use the same example as for Theorem 1). The scalar-valued case $q=\infty$ is due to Brudnyi and Ganzburg [BG]. Nazarov, Sodin and Volberg [NSV] have obtained Theorem 2 independently by somewhat different methods. In $\S 6$, Remark 2 below, we shall show how one can obtain the case $r \leq 1$ and general $q$ in Theorem 1 from Theorem 2.

In common with Bobkov's work [Bobk] (and that of Nazarov, Sodin and Volberg $[\mathrm{NSV}]$ ) the main tool in this current work is the utilisation of a certain powerful extremal result of Kannan, Lovász and Simonovits which we now state.

For $a, b \in \mathbb{R}^{n}$ and $\lambda \geq 1$ define the measures $\mu_{a, b, \lambda}$ by $\left\langle\phi, \mu_{a, b, \lambda}\right\rangle=\int_{0}^{1} \phi(a(1-$ $t)+b t)(\lambda-t)^{n-1} d t$.

Theorem ([KLS]). Suppose $f_{1}, f_{2}, f_{3}, f_{4}$ are continuous nonnegative integrable functions on $\mathbb{R}^{n}$ and $\alpha, \beta>0$. Suppose that for every $a, b \in \mathbb{R}^{n}$ and $\lambda \geq 1$,

$$
\left(\int f_{1} d \mu_{a, b, \lambda}\right)^{\alpha}\left(\int f_{2} d \mu_{a, b, \lambda}\right)^{\beta} \leq\left(\int f_{3} d \mu_{a, b, \lambda}\right)^{\alpha}\left(\int f_{4} d \mu_{a, b, \lambda}\right)^{\beta} .
$$

Then for every convex open set $K$ in $\mathbb{R}^{n}$

$$
\left(\int_{K} f_{1}\right)^{\alpha}\left(\int_{K} f_{2}\right)^{\beta} \leq\left(\int_{K} f_{3}\right)^{\alpha}\left(\int_{K} f_{4}\right)^{\beta} .
$$

(Note that the reverse implication is straightforward.)

Finally, $C$ will denote a generic absolute constant whose precise value may change from line to line.

\section{Reduction to weighted inequalities in dimension 1}

We shall first prove the results in the scalar-valued setting and then show in $\S 5$ how one can extend the arguments to the vector-valued setting. In the scalar-valued setting, by the Kannan, Lovász and Simonovits theorem of the introduction, Theorems 1 and 2 are equivalent (after a limiting argument because $\chi_{\{x \in K:|p(x)| \leq \alpha\}}$ is not a continuous function) to Theorems 3 and 4 respectively: 
Theorem 3. Let $p: \mathbb{R} \rightarrow \mathbb{C}$ be a polynomial of degree at most $d, n \in \mathbb{N}, \lambda \geq 1$ and $0 \leq r \leq q \leq \infty$. Then there exists an absolute constant $C$ independent of the above parameters such that

$$
\left(\frac{\int_{0}^{1}|p(t)|^{\frac{q}{d}}(\lambda-t)^{n-1} d t}{\int_{0}^{1}(\lambda-t)^{n-1} d t}\right)^{\frac{1}{q}} \leq C \frac{[n B(n, q+1)]^{\frac{1}{q}}}{[n B(n, r+1)]^{\frac{1}{r}}}\left(\frac{\int_{0}^{1}|p(t)|^{\frac{r}{d}}(\lambda-t)^{n-1} d t}{\int_{0}^{1}(\lambda-t)^{n-1} d t}\right)^{\frac{1}{r}} .
$$

Theorem 4. Let Let $p: \mathbb{R} \rightarrow \mathbb{C}$ be a polynomial of degree at most $d, n \in \mathbb{N}$, $\lambda \geq 1$ and $0 \leq q \leq \infty$. Then there exists an absolute constant $C$ independent of the above parameters so that for any $\alpha>0$,

$$
\begin{gathered}
\left(\frac{\int_{0}^{1}|p(t)|^{\frac{q}{d}}(\lambda-t)^{n-1} d t}{\int_{0}^{1}(\lambda-t)^{n-1} d t}\right)^{\frac{1}{q}} \frac{\alpha^{-\frac{1}{d}} \int_{0}^{1} \chi_{\{|p(t)| \leq \alpha\}}(\lambda-t)^{n-1} d t}{\int_{0}^{1}(\lambda-t)^{n-1} d t} \\
\leq C n(n B(n, q+1))^{\frac{1}{q}} .
\end{gathered}
$$

Although the forms of the inequalities in Theorems 3 and 4 make sense only for $0<r \leq q<\infty$, it is clear how to extend them when $r=0, q=0$ and/or $r=\infty, q=\infty$. For instance, when $q=0$, the conclusion of Theorem 4 takes the form

$$
\exp \frac{1}{d}\left(\frac{\int_{0}^{1}[\log |p(t)|](\lambda-t)^{n-1} d t}{\int_{0}^{1}(\lambda-t)^{n-1} d t}\right) \frac{\alpha^{-\frac{1}{d}} \int_{0}^{1} \chi_{\{|p(t)| \leq \alpha\}}(\lambda-t)^{n-1} d t}{\int_{0}^{1}(\lambda-t)^{n-1} d t} \leq C .
$$

To prove Theorems 3 and 4, we may of course assume that $r>0$ and $q<\infty$ and then pass to the limit.

\section{Proof of Theorem 3}

We begin with the proof of Theorem 3 . We first need some preliminary lemmas. The first is a well-known elementary Remez type inequality. It is also a simple consequence of the case $n=1$ of Theorem 1 or Theorem 3 and as such is already contained in $[\mathrm{BG}]$, for instance. We include a simple proof for the convenience of the reader.

Lemma 1. There is an absolute constant $C$ so that if $p: \mathbb{R} \rightarrow \mathbb{C}$ is a polynomial of degree at most $d$, if $0 \leq r \leq q \leq \infty$, and if $t \geq u$, then

$$
\left(\frac{1}{t} \int_{0}^{t}|p|^{\frac{q}{d}}\right)^{\frac{1}{q}} \leq C \frac{t}{u}\left(\frac{1}{u} \int_{0}^{u}|p|^{\frac{r}{d}}\right)^{\frac{1}{r}} .
$$

(We have the usual interpretation in the limiting cases $r, q=0, \infty$.) 
Proof of Lemma 1. We may assume that $q=\infty, r=0$ and $u=1$. So we want to show

$$
\left\||p|^{\frac{1}{d}}\right\|_{L^{\infty}[0, t]} \leq C t \exp \frac{1}{d} \int_{0}^{1} \log |p(s)| d s
$$

for $t \geq 1$. Clearly we may also assume that $p(z)=\prod\left(z-\zeta_{j}\right)$ is monic. Now

$$
\max _{0 \leq s \leq t}|p(s)|^{\frac{1}{d}}=\max _{0 \leq s \leq t} \prod\left|s-\zeta_{j}\right|^{\frac{1}{d}}=\max _{0 \leq s \leq 1} \prod\left|s t-\zeta_{j}\right|^{\frac{1}{d}} \leq t \max _{0 \leq s \leq 1} \prod\left|s-\zeta_{j} / t\right|^{\frac{1}{d}} .
$$

Moreover $t \geq 1$ and $\left|\zeta_{j}\right| \geq 2$ implies $\left|s-\zeta_{j} / t\right| \leq 2\left|\zeta_{j}\right| \leq 4\left|s-\zeta_{j}\right|$ for $0 \leq s \leq 1$; so that we are left with proving

$$
\max _{0 \leq s \leq 1} \prod_{\left|\zeta_{j}\right| \leq 2}\left|s-\zeta_{j} / t\right|^{\frac{1}{d}} \leq C \exp \left\{\frac{1}{d} \int_{0}^{1} \sum_{\left|\zeta_{j}\right| \leq 2} \log \left|s-\zeta_{j}\right| d s\right\} .
$$

The term on the left of this inequality is bounded by 3 , while the term on the right is bounded below by $\exp \gamma$ where $\gamma=\inf _{|\zeta| \leq 2} \int_{0}^{1} \log |s-\zeta| d s$. The lemma is established with $C=12 \mathrm{e}^{-\gamma}$.

Lemma 2. There is an absolute constant $C$ so that if $0<r \leq 2 m$,

$$
\left(\int_{0}^{\frac{r}{2}}(1-t / m)^{m-1} t^{r+1} d t\right)^{\frac{1}{r}} \geq C\left(\int_{0}^{m}(1-t / m)^{m-1} t^{r+1} d t\right)^{\frac{1}{r}} .
$$

We remark that the term on the right hand side of Lemma 2 is itself bounded below by $m[(m+1) B(m+1, r+1)]^{\frac{1}{r}}$.

Proof of Lemma 2.

$$
\begin{aligned}
\int_{0}^{\frac{r}{2}}(1-t / m)^{m-1} t^{r+1} d t \geq \int_{0}^{\frac{r}{4}}(1-t / m)^{m-1} t^{r+1} d t \geq & \int_{0}^{\frac{r}{4}} \mathrm{e}^{-2 t} t^{r+1} d t \\
& \geq \frac{1}{r+2}\left(\frac{r}{4}\right)^{r+2} \mathrm{e}^{-\frac{r}{2}}
\end{aligned}
$$

But

$$
\begin{aligned}
\int_{0}^{m}(1-t / m)^{m-1} t^{r+1} d t \leq(r+1) \int_{0}^{\infty}(1-t / m)^{m} t^{r} d t & \\
& \leq(r+1) \int_{0}^{\infty} \mathrm{e}^{-t} t^{r} d t=(r+1) !
\end{aligned}
$$

Taking $r^{\prime}$ th roots establishes the lemma.

Lemma 3. There is an absolute constant $C$ so that if $p: \mathbb{R} \rightarrow \mathbb{C}$ is a polynomial of degree at most $d$, if $0<r \leq q<\infty$ and if $\frac{r}{2} \leq t \leq x$, then

$$
\int_{0}^{t}|p|^{\frac{q}{d}} \leq \frac{C^{q} t^{q+1}}{m^{q}[(m+1) B(m+1, r+1)]^{\frac{q}{r}}}\left[\int_{0}^{x}(1-u / m)^{m-1}\left(\int_{0}^{u}|p|^{\frac{r}{d}}\right) d u\right]^{\frac{q}{r}} .
$$


Proof. By Lemma 1, we have for $t \geq u$

$$
u^{r+1}\left(\int_{0}^{t}|p|^{\frac{q}{d}}\right)^{\frac{r}{q}} \leq C^{r} t^{r+\frac{r}{q}} \int_{0}^{u}|p|^{\frac{r}{d}} .
$$

Multiplying this inequality by $(1-u / m)^{m-1}$ and integrating with respect to $u$ from 0 to $t$ yields

$$
\left[\int_{0}^{t} u^{r+1}(1-u / m)^{m-1} d u\right]\left(\int_{0}^{t}|p|^{\frac{q}{d}}\right)^{\frac{r}{q}} \leq C^{r} t^{r+\frac{r}{q}} \int_{0}^{t}(1-u / m)^{m-1}\left(\int_{0}^{u}|p|^{\frac{r}{d}}\right) d u .
$$

Lemma 2 and the remark following its statement now imply that

$$
\left(\int_{0}^{t}|p|^{\frac{q}{d}}\right)^{\frac{r}{q}} \leq \frac{C^{r} t^{r+\frac{r}{q}}}{m^{r}[(m+1) B(m+1, r+1)]} \int_{0}^{t}(1-u / m)^{m-1}\left(\int_{0}^{u}|p|^{\frac{r}{d}}\right) d u .
$$

Lemma 3 now follows upon taking $\frac{q}{r}$ 'th roots.

Proof of Theorem 3. We may assume that $0<r \leq q<\infty$. For ease of notation we write $m$ for $n-1$, and denote $[(m+1) B(m+1, q+1)]^{\frac{1}{q}}$ by $A_{q}$ (for $m$ fixed). We assume $m \geq 2$ (otherwise the proof simplifies), and changing variables we see that we have to show, for each $\lambda \geq 1$ and all polynomials $p$ of degree at most $d$

$$
\left(\frac{\int_{0}^{\frac{m}{\lambda}}|p(t)|^{\frac{q}{d}}(1-t / m)^{m} d t}{\int_{0}^{\frac{m}{\lambda}}(1-t / m)^{m} d t}\right)^{\frac{1}{q}} \leq C \frac{A_{q}}{A_{r}}\left(\frac{\int_{0}^{\frac{m}{\lambda}}|p(t)|^{\frac{r}{d}}(1-t / m)^{m} d t}{\int_{0}^{\frac{m}{\lambda}}(1-t / m)^{m} d t}\right)^{\frac{1}{r}} .
$$

Case 1: $\mathrm{m} \leq \lambda$

Notice that if $0 \leq t \leq 1, \mathrm{e}^{-2} \leq(1-t / m)^{m} \leq 1$ for $m \geq 2$. Moreover,

$$
\left(\frac{\lambda}{m} \int_{0}^{\frac{m}{\lambda}}|p(t)|^{\frac{q}{d}} d t\right)^{\frac{1}{q}} \leq C\left(\frac{\lambda}{m} \int_{0}^{\frac{m}{\lambda}}|p(t)|^{\frac{r}{d}} d t\right)^{\frac{1}{r}}
$$

for $0<q, r<\infty$ by Lemma 1 . Finally, since $A_{q}$ is an increasing function of $q$ this case is complete.

Case 2: $m>\lambda$.

Let $x=\frac{m}{\lambda}$; then $1 \leq x \leq m$ as $\lambda \geq 1$. For $1 \leq x \leq m, \int_{0}^{x}(1-t / m)^{m} d t$ is bounded above and below by absolute constants. So we wish to see that for $1 \leq x \leq m$ and $0<r \leq q$,

$$
\left(\int_{0}^{x}|p|^{\frac{q}{d}}(1-t / m)^{m} d t\right)^{\frac{1}{q}} \leq C \frac{A_{q}}{A_{r}}\left(\int_{0}^{x}|p|^{\frac{r}{d}}(1-t / m)^{m} d t\right)^{\frac{1}{r}} .
$$

Now

$$
\int_{0}^{x}|p|^{\frac{q}{d}}(1-t / m)^{m} d t=\int_{0}^{x}(1-t / m)^{m-1}\left(\int_{0}^{t}|p|^{\frac{q}{d}}\right) d t+(1-x / m)^{m} \int_{0}^{x}|p|^{\frac{q}{d}} .
$$

We shall concentrate on the first term, the arguments for the second being similar but easier. We distinguish two subcases of (1): 
Subcase (i): $r / 2 \leq x$.

In this subcase, $r \leq 2 m$ and

$$
\begin{aligned}
\int_{0}^{x}(1-t / m)^{m-1}\left(\int_{0}^{t}|p|^{\frac{q}{d}}\right) d t & \\
& =\int_{\frac{r}{2}}^{x}(1-t / m)^{m-1}\left(\int_{0}^{t}|p|^{\frac{q}{d}}\right) d t+\int_{0}^{\frac{r}{2}}(1-t / m)^{m-1}\left(\int_{0}^{t}|p|^{\frac{q}{d}}\right) d t .
\end{aligned}
$$

The estimate for the second term here is a special case $(x=r / 2)$ of subcase (ii) below, so it suffices to deal with the first term. Using Lemma 3 we have

$$
\begin{aligned}
\int_{\frac{r}{2}}^{x}\left(1-\frac{t}{m}\right)^{m-1}\left(\int_{0}^{t}|p|^{\frac{q}{d}}\right) d t \\
\leq \frac{C^{q}}{m^{q} A_{r}^{q}}\left[\int_{\frac{r}{2}}^{x}\left(1-\frac{t}{m}\right)^{m-1} t^{q+1} d t\right]\left[\int_{0}^{x}\left(1-\frac{u}{m}\right)^{m-1}\left(\int_{0}^{u}|p|^{\frac{r}{d}}\right) d u\right]^{\frac{q}{r}} \\
\leq \frac{C^{q}(q+1) m^{q+1} A_{q}^{q}}{m^{q} A_{r}^{q}(m+1)}\left(\int_{0}^{x}|p|^{\frac{r}{d}}\left(1-\frac{t}{m}\right)^{m} d t\right)^{\frac{q}{r}} .
\end{aligned}
$$

Taking $q$ 'th roots establishes subcase (i).

Subcase (ii): $1 \leq x \leq r / 2$.

$$
\begin{aligned}
& \int_{0}^{x}(1-t / m)^{m-1}\left(\int_{0}^{t}|p|^{\frac{q}{d}}\right) d t= \\
& \qquad \int_{0}^{1}(1-t / m)^{m-1}\left(\int_{0}^{t}|p|^{\frac{q}{d}}\right) d t+\int_{1}^{x}(1-t / m)^{m-1}\left(\int_{0}^{t}|p|^{\frac{q}{d}}\right) d t .
\end{aligned}
$$

The first term is easy to deal with since by Lemma 1

$$
\begin{aligned}
\int_{0}^{1}(1-t / m)^{m-1} \int_{0}^{t}|p|^{\frac{q}{d}} d t \leq \int_{0}^{1}|p|^{\frac{q}{d}} \leq & C^{q}\left(\int_{0}^{1}|p|^{\frac{r}{d}}\right)^{\frac{q}{r}} \\
& \leq C^{q}\left(\int_{0}^{1}|p|^{\frac{r}{d}}(1-t / m)^{m-1} d t\right)^{\frac{q}{r}} .
\end{aligned}
$$

For the second term, Lemma 1 implies that for $t \geq u$

$$
u^{r+1}\left(\int_{0}^{t}|p|^{\frac{q}{d}}\right)^{\frac{r}{q}} \leq C^{r} t^{r+\frac{r}{q}} \int_{0}^{u}|p|^{\frac{r}{d}} .
$$

Multiplying this inequality by $(1-u / m)^{m-1}$ and integrating with respect to $u$ from 0 to $t$ yields

$$
\begin{gathered}
{\left[\int_{0}^{t} u^{r+1}(1-u / m)^{m-1} d u\right]\left[\int_{0}^{t}|p|^{\frac{q}{d}}\right]^{\frac{r}{q}} \leq C^{r} t^{r+\frac{r}{q}} \int_{0}^{t}\left(\int_{0}^{u}|p|^{\frac{r}{d}}\right)(1-u / m)^{m-1} d u} \\
\leq C^{r} t^{r+\frac{r}{q}} \int_{0}^{t}|p|^{\frac{r}{d}}(1-u / m)^{m} d u \leq C^{r} t^{r+\frac{r}{q}} \int_{0}^{x}|p|^{\frac{r}{d}}(1-u / m)^{m} d u
\end{gathered}
$$


provided $t \leq x$. But

$$
\int_{0}^{t} u^{r+1}(1-u / m)^{m-1} d u \geq \mathrm{e}^{-t} \int_{0}^{\frac{t}{2}} u^{r+1} d u=\frac{\mathrm{e}^{-t} t^{r+2}}{2^{r+2}(r+2)}
$$

Thus for $1 \leq t \leq x \leq \frac{r}{2}$,

$$
\int_{0}^{t}|p|^{\frac{q}{d}} \leq C^{q} \mathrm{e}^{\frac{t q}{r}} t^{1-\frac{2 q}{r}}\left[\int_{0}^{x}|p|^{\frac{r}{d}}(1-u / m)^{m} d u\right]^{\frac{q}{r}} \leq C^{q}\left[\int_{0}^{x}|p|^{\frac{r}{d}}(1-u / m)^{m} d u\right]^{\frac{q}{r}} .
$$

Now multiplying both sides of this inequality by $(1-t / m)^{m-1}$ and integrating with respect to $t$ from 1 to $x$ gives

$$
\begin{gathered}
\int_{1}^{x}(1-t / m)^{m-1}\left(\int_{0}^{t}|p|^{\frac{q}{d}}\right) d t \leq C^{q}\left[\int_{1}^{x}(1-t / m)^{m-1} d t\right]\left[\int_{1}^{x}|p|^{\frac{r}{d}}(1-u / m)^{m} d u\right]^{\frac{q}{r}} \\
\leq C^{q}\left[\int_{0}^{x}|p|^{\frac{r}{d}}(1-u / m)^{m} d u\right]^{\frac{q}{r}}
\end{gathered}
$$

(as $(1-t / m)^{m-1} \leq 1$ and $\left.x \leq \frac{r}{2} \leq q\right)$. Taking $q^{\prime}$ th roots finishes subcase (ii) of (2), and hence (1), proving Theorem 3.

\section{Proof of Theorem 4}

The first step in proving Theorem 4 is the special case $n=1, q=\infty$ :

Lemma 4. There is an absolute constant $C$ so that for all polynomials $p: \mathbb{R} \rightarrow$ $\mathbb{C}$ of degree at most $d$ and all intervals $I$,

$$
\|p\|_{L^{\infty}(I)}^{\frac{1}{d}} \alpha^{-\frac{1}{d}}|\{x \in I:|p(x)| \leq \alpha\}| \leq C|I| .
$$

This lemma is an old result and in fact the best constant $C$ is known to be 4 . This is due to Dudley and Randol, [DR]. However this result for some absolute constant $C$ is an easy consequence of a classical inequality of $\mathrm{H}$. Cartan $[\mathrm{C}]$ which we now state:

Cartan's lemma. Let $w_{1}, w_{2}, \ldots, w_{d}$ be $d$ points in the complex plane $\mathbb{C}$ and let $h>0$. Then the set of points $z \in \mathbb{C}$ such that the inequality

$$
\prod_{j=1}^{d}\left|z-w_{j}\right| \leq h^{d}
$$

holds can be covered by at most d circles, the sum of whose radii is $2 \mathrm{eh}$.

Note, in particular, Cartan's lemma implies the corresponding statement of Lemma 4 for monic (as opposed to $L^{\infty}$ - normalised) polynomials. We provide a proof of Lemma 4 for completeness.

Proof of Lemma 4. We may assume that $I=[0,1]$ by translating and dilating the polynomial $p$. Observe that the statement of the lemma is invariant under multiplication of $p$ by any nonzero constant, and (up to changing the value of $C$ ) under multiplication of $p$ by a function, whose $d$ 'th root is bounded above and 
below by absolute constants. So if $p(z)=A \prod\left(z-\zeta_{j}\right)$, we may multiply $p$ by $\prod_{\left|\zeta_{j}\right| \geq 2}\left|\zeta_{j}\right|\left(z-\zeta_{j}\right)^{-1}$ and then by $\left(A \prod_{\left|\zeta_{j}\right| \geq 2}\left|\zeta_{j}\right|\right)^{-1}$ without changing matters. Thus we may assume that $p(z)=\prod_{\left|\zeta_{j}\right| \leq 2}\left(z-\zeta_{j}\right)$. This modified $p(z)$ is now monic, has degree $k \leq d$ say, and when restricted to the unit interval $[0,1]$ satisfies $\|p\|_{\infty}^{\frac{1}{d}} \leq 3$. We may therefore assume $\alpha \leq 1$ and Cartan's inequality tells us that

$$
|\{x \in[0,1]:|p(x)| \leq \alpha\}| \leq C \alpha^{\frac{1}{k}} \leq C \alpha^{\frac{1}{d}},
$$

completing the proof of the lemma.

Note the case $q=\infty$ of Theorem 4 and thus Theorem 2 is now an immediate consequence: we merely have to observe that for $0 \leq t \leq 1$ and $\lambda \geq 1$, we have $(\lambda-t)^{n-1} \leq n \int_{0}^{1}(\lambda-s)^{n-1} d s$.

Proof of Theorem 4. Again we may assume that $0<q<\infty$. For ease of notation we again write $m$ for $n-1$ and assume $m \geq 2$ (the cases $m=0$ and $m=1$ are easier). Let

$$
I^{q}=\frac{\int_{0}^{1}|p(t)|^{\frac{q}{d}}(\lambda-t)^{m} d t}{\int_{0}^{1}(\lambda-t)^{m} d t} \quad \text { and } \quad I I=\frac{\alpha^{-\frac{1}{d}} \int_{0}^{1} \chi_{\{|p| \leq \alpha\}}(\lambda-t)^{m} d t}{\int_{0}^{1}(\lambda-t)^{m} d t} .
$$

We wish to show that $I \cdot I I \leq C(m+1)[(m+1) B(m+1, q+1)]^{\frac{1}{q}}$. We immediately make the change of variables $t \rightarrow \frac{\lambda}{m} t$ in all integrals, so that

$$
I^{q}=\frac{\int_{0}^{\frac{m}{\lambda}}|p(t)|^{\frac{q}{d}}\left(1-\frac{t}{m}\right)^{m} d t}{\int_{0}^{\frac{m}{\lambda}}\left(1-\frac{t}{m}\right)^{m} d t} \quad \text { and } \quad I I=\frac{\alpha^{-\frac{1}{d}} \int_{0}^{\frac{m}{\lambda}} \chi_{\{|p| \leq \alpha\}}\left(1-\frac{t}{m}\right)^{m} d t}{\int_{0}^{\frac{m}{\lambda}}\left(1-\frac{t}{m}\right)^{m} d t}
$$

(for a possibly different polynomial $p$ ). Note that if $D:=\int_{0}^{\frac{m}{\lambda}}\left(1-\frac{t}{m}\right)^{m} d t$, then for $m \leq \lambda$, we have $D \geq \int_{0}^{\frac{m}{\lambda}}(1-1 / m)^{m} d t \geq \frac{m}{\lambda} \frac{1}{2 \mathrm{e}}$ while for $m \geq \lambda$, $D \geq \int_{0}^{1}(1-t / m)^{m} d t \geq \int_{0}^{1}(1-1 / m)^{m} d t \geq \frac{1}{2 \mathrm{e}}$.

Case 1: $m \leq \lambda$.

In this case we have

$$
I^{q} \leq \frac{2 \mathrm{e} \lambda}{m} \int_{0}^{\frac{m}{\lambda}}|p(t)|^{\frac{q}{d}}(1-t / m)^{m} d t \leq \frac{2 \mathrm{e} \lambda}{m} \int_{0}^{\frac{m}{\lambda}}|p(t)|^{\frac{q}{d}} d t,
$$


while

$$
I I \leq \frac{2 \mathrm{e} \lambda}{m} \alpha^{-\frac{1}{d}} \int_{0}^{\frac{m}{\lambda}} \chi_{\{|p| \leq \alpha\}}(1-t / m)^{m} d t \leq \frac{2 \mathrm{e} \lambda}{m} \alpha^{-\frac{1}{d}} \int_{0}^{\frac{m}{\lambda}} \chi_{\{|p| \leq \alpha\}} d t
$$

so that

$$
I \cdot I I \leq(2 \mathrm{e})^{1+\frac{1}{q}}\left(\frac{\lambda}{m} \int_{0}^{\frac{m}{\lambda}}|p(t)|^{\frac{q}{d}} d t\right)^{\frac{1}{q}} \cdot \alpha^{-\frac{1}{d}} \frac{\lambda}{m} \int_{0}^{\frac{m}{\lambda}} \chi_{\{|p| \leq \alpha\}} d t \leq C(2 \mathrm{e})^{1+\frac{1}{q}}
$$

by Lemma 4 . Thus $I \cdot I I$ is bounded above by an absolute constant in this case.

Case 2: $m>\lambda$.

In this case, since $D$ is uniformly bounded below and the numerators of $I$ and II are decreasing with $\lambda$, we may take $\lambda=1$ and reduce matters to showing that

$$
\widetilde{I} \cdot \widetilde{I I} \leq C(m+1)[(m+1) B(m+1, q+1)]^{\frac{1}{q}}
$$

where

$\widetilde{I}^{q}=\int_{0}^{m} \mid p(t)^{\frac{q}{d}}(1-t / m)^{m} d t \quad$ and $\quad \widetilde{I I}=\alpha^{-\frac{1}{d}} \int_{0}^{m} \chi_{\{|p| \leq \alpha\}}(1-t / m)^{m} d t$.

Now

$\widetilde{I}^{q}=\int_{0}^{m}(1-t / m)^{m} \frac{d}{d t}\left\{\int_{0}^{t}|p(s)|^{\frac{q}{d}} d s\right\} d t=\int_{0}^{m}(1-t / m)^{m-1}\left\{\int_{0}^{t}|p(s)|^{\frac{q}{d}} d s\right\} d t$ which in turn is less than $\int_{0}^{1} H d t+C^{q} H \int_{1}^{m}(1-t / m)^{m-1} t^{q+1} d t$ where $H=$ $\int_{0}^{1}|p(s)|^{\frac{q}{d}} d s$, by Lemma 1 . Hence

$$
\begin{gathered}
\widetilde{I}^{q} \leq H\left[1+C^{q} \int_{1}^{m}(1-t / m)^{m-1} t^{q+1} d t\right] \leq H\left[1+C^{q} m^{q+2} \int_{0}^{1}(1-s)^{m-1} s^{q+1} d s\right] \\
=H\left[1+C^{q} m^{q+1}(q+1) B(m+1, q+1)\right] .
\end{gathered}
$$

Therefore $\widetilde{I} \leq C H^{\frac{1}{q}}(m+1)[(m+1) B(m+1, q+1)]^{\frac{1}{q}}$. On the other hand,

$$
\begin{aligned}
\widetilde{I I}= & \alpha^{-\frac{1}{d}} \int_{0}^{m} \chi_{\{|p| \leq \alpha\}}(1-t / m)^{m} d t=\alpha^{-\frac{1}{d}} \int_{0}^{m}(1-t / m)^{m-1} \int_{0}^{t} \chi_{\{|p(s)| \leq \alpha\}} d s d t \\
\leq & \alpha^{-\frac{1}{d}}\left[\int_{0}^{1} \int_{0}^{1} \chi_{\{|p(s)| \leq \alpha\}} d s d t+\int_{1}^{m}(1-t / m)^{m-1} \int_{0}^{t} \chi_{\{|p(s)| \leq \alpha\}} d s d t\right] \\
& \leq \frac{C}{K}+\frac{C}{K} \int_{1}^{m}(1-t / m)^{m-1} t d t \leq \frac{C}{K} \int_{0}^{\infty} \mathrm{e}^{-t / 2} t d t \leq \frac{C}{K}
\end{aligned}
$$

by Lemma 4 , where $K=\left\||p|^{\frac{1}{d}}\right\|_{L^{\infty}[0,1]}$. Thus

$$
\widetilde{I} \cdot \widetilde{I I} \leq C\left[\frac{\left\||p|^{\frac{1}{d}}\right\|_{L^{q}[0,1]}}{\left\|\left.p\right|^{\frac{1}{d}}\right\|_{L^{\infty}[0,1]}}\right](m+1)[(m+1) B(m+1, q+1)]^{\frac{1}{q}}
$$


which in turn is less than $C(m+1)[(m+1) B(m+1, q+1)]^{\frac{1}{q}}$ as required, completing the proof of Theorem 4. (Note that we have used in passing that $n[n B(n, q+1)]^{\frac{1}{q}}$ is bounded below uniformly in $n$ and $\left.q.\right)$

\section{The vector-valued case}

To extend Theorems 1 and 2 to the vector-valued setting, we first observe that our arguments extend to a wider class of functions than polynomials of degree at most $d$. Following a preliminary version of [NSV], we say that a function $u$ : $\mathbb{R}^{n} \rightarrow \mathbb{R}$ is of class $\mathcal{L}$ if it is the restriction to $\mathbb{R}^{n}$ of a plurisubharmonic function $\widetilde{u}: \mathbb{C}^{n} \rightarrow \mathbb{R}$ such that $\limsup \frac{\tilde{u}(z)}{\log |z|} \leq 1$. When $n=1, u(x)=\frac{1}{d} \log |p(x)|$ is of class $\mathcal{L}$ if $p: \mathbb{R} \rightarrow \mathbb{C}$ is a polynomial of degree $d$. We can write such a $p$ as $p(x)=A \prod_{j=1}^{d}\left(x-\zeta_{j}\right)$, so that $\frac{1}{d} \log |p(x)|=\frac{1}{d} \log |A|+\frac{1}{d} \sum_{j=1}^{d} \log \left|x-\zeta_{j}\right|$, and the distinguishing feature of a function of class $\mathcal{L}$ (when $n=1$ ) is that it can be written as $u(x)=$ constant $+\int \log |x-\zeta| d \mu(\zeta)$ where $\mu$ is a positive measure of mass at most one in the plane. This is the well-known Riesz representation for subharmonic functions, see for example Hayman's book [H]. In particular, it is not difficult to see that the key lemmas, Lemma 1 and Lemma 4, remain valid if one replaces $|p(x)|^{\frac{1}{d}}$ with $\exp u(x)$, where $u$ is a general function of class $\mathcal{L}$ in one dimension. With these remarks in mind the reader will have no trouble extending Theorems 1 and 2 to functions of class $\mathcal{L}$ to obtain the following:

Theorem 5. Let $u: \mathbb{R}^{n} \rightarrow \mathbb{R}$ be a function of class $\mathcal{L}, 0 \leq r \leq q \leq \infty$ and $K$ be a convex body in $\mathbb{R}^{n}$ of volume 1 . Then there exists an absolute constant $C$ independent of $r, q, K, n$ and $u$ so that

$$
\left\|\mathrm{e}^{u}\right\|_{L^{q}(K)} \leq C \frac{[n B(n, q+1)]^{\frac{1}{q}}}{[n B(n, r+1)]^{\frac{1}{r}}}\left\|\mathrm{e}^{u}\right\|_{L^{r}(K)} .
$$

Theorem 6. Let $u: \mathbb{R}^{n} \rightarrow \mathbb{R}$ be a function of class $\mathcal{L}, 0 \leq q \leq \infty$ and $K$ be a convex body in $\mathbb{R}^{n}$ of volume 1 . Then there exists an absolute constant $C$ independent of $q, K, n$ and $u$ so that

$$
\left\|\mathrm{e}^{u}\right\|_{L^{q}(K)}\left\|\mathrm{e}^{-u}\right\|_{L^{1, \infty}(K)} \leq C n[n B(n, q+1)]^{\frac{1}{q}} .
$$

To obtain the vector-valued extension of Theorems 1 and 2, we simply observe that whenever $p: \mathbb{R}^{n} \rightarrow X$ is a polynomial of degree at most $d$ with values in a Banach space $X, u(x)=\frac{1}{d} \log \|p(x)\|$ is a function of class $\mathcal{L}$. Indeed, the estimate $\limsup _{|z| \rightarrow \infty} \frac{\tilde{u}(z)}{\log |z|} \leq 1$ is straightforward, and using the fact $\|w\|=$ $\sup _{X^{*},\|\ell\| \leq 1}|\ell(w)|$ for any $w \in X$, one easily sees that $\widetilde{u}(z)$ is plurisubharmonic. 


\section{Further remarks}

1. If we let $m \rightarrow \infty$ in inequality (1), we have, since $(1-t / m)^{m} \leq \mathrm{e}^{-t}$ for $m>0$ and $0<t \leq m$,

$$
\left(\int_{0}^{x}|p(t)|^{\frac{q}{d}} \mathrm{e}^{-t} d t\right)^{\frac{1}{q}} \leq C \frac{\max (q, 1)}{\max (r, 1)}\left(\int_{0}^{x}|p(t)|^{\frac{r}{d}} \mathrm{e}^{-t} d t\right)^{\frac{1}{r}}
$$

by the dominated convergence theorem, where $C$ is absolute: combining this with Lemmas 1 and 4 yields the following results.

Proposition 1. There exists an absolute constant $C$ such that if $p$ is a polynomial of degree at most $d, N>0$, and $0<r \leq q<\infty$,

$$
\left(\frac{\int_{0}^{N}|p(t)|^{\frac{q}{d}} \mathrm{e}^{-t} d t}{\int_{0}^{N} \mathrm{e}^{-t} d t}\right)^{\frac{1}{q}} \leq C \frac{\max (q, 1)}{\max (r, 1)}\left(\frac{\int_{0}^{N}|p(t)|^{\frac{r}{d}} \mathrm{e}^{-t} d t}{\int_{0}^{N} \mathrm{e}^{-t} d t}\right)^{\frac{1}{r}}
$$

Proposition 2. There exists an absolute constant $C$ such that if $p$ is a polynomial of degree at most $d, N>0$, and $0<q<\infty$,

$$
\left(\frac{\int_{0}^{N}|p(t)|^{\frac{q}{d}} \mathrm{e}^{-t} d t}{\int_{0}^{N} \mathrm{e}^{-t} d t}\right)^{\frac{1}{q}} \cdot \frac{\alpha^{-\frac{1}{d}} \int_{0}^{N} \chi_{\{|p(t)| \leq \alpha\}} \mathrm{e}^{-t} d t}{\int_{0}^{N} \mathrm{e}^{-t} d t} \leq C \max (q, 1) .
$$

Propositions 1 and 2 are also true if one replaces $|p(t)|^{\frac{1}{d}}$ with $\exp u$, where $u$ is any function of class $\mathcal{L}$. Using another theorem of Kannan, Lovász and Simonovits [KLS] (which is similar to their theorem stated in the introduction except that the measures $\mu$ are replaced by measures with exponential densities) we then obtain

Theorem 7. Let $X$ be a Banach space and let $p: \mathbb{R}^{n} \rightarrow X$ be a polynomial of degree at most $d$. Suppose $0<r \leq q<\infty$ and $\mu$ is a log-concave probability measure on $\mathbb{R}^{n}$. Then there is an absolute constant $C$ such that

$$
\left(\int\|p(x)\|^{\frac{q}{d}} d \mu(x)\right)^{\frac{1}{q}} \leq C \frac{\max (q, 1)}{\max (r, 1)}\left(\int\|p(x)\|^{\frac{r}{d}} d \mu(x)\right)^{\frac{1}{r}},
$$

and for the sublevel set estimate:

Theorem 8. There exists an absolute constant $C$ such that if $p: \mathbb{R}^{n} \rightarrow X$ is a polynomial of degree at most $d, 0<q<\infty$, and $\mu$ is a log-concave probability measure on $\mathbb{R}^{n}$, then

$$
\left(\int\|p(x)\|^{\frac{q}{d}} d \mu(x)\right)^{\frac{1}{q}} \cdot \alpha^{-\frac{1}{d}} \mu\left\{x \in \mathbb{R}^{n}:\|p(x)\| \leq \alpha\right\} \leq C q .
$$


A measure is said to be log-concave if it is supported by an affine subspace $L$ of $\mathbb{R}^{n}$, and with respect to Lebesgue measure on $L$ has a density of the form $\mathrm{e}^{-g(x)}$ where the set $K=\{x: g(x)<\infty\}$ and $\left.g\right|_{K}$ are convex. In addition to characteristic functions of convex bodies, these measures include gaussians $\mathrm{e}^{-|x|^{2}} d x$. Of course we can let $q$ or $r \rightarrow 0$ in Theorems 7 and 8 to obtain estimates in the $\exp -\log$ class $L^{0}$.

2. To see why Theorem 2 implies the case $r \leq 1$ and general $q$ of Theorem 1, we first observe that Theorem 2 has a trivial reverse inequality. Considering the sublevel set for $\|p(x)\|$ with $\alpha^{\frac{q}{d}}=2 \int_{K}\|p\|^{\frac{q}{d}}$, we have $1 / 4 \leq\|\| p\left\|^{\frac{1}{d}}\right\|_{L^{q}(K)}$. $\sup _{\alpha>0} \alpha^{-\frac{1}{d}}|\{x \in K:\|p(x)\| \leq \alpha\}|$ uniformly for $q>0$. Hence, by Theorem 2

$$
1 / 4 \leq\|\| p\left\|^{\frac{1}{d}}\right\|_{L^{q}(K)} \cdot \sup _{\alpha>0} \alpha^{-\frac{1}{d}}|\{x \in K:\|p(x)\| \leq \alpha\}| \leq C n(n B(n, q+1))^{\frac{1}{q}} .
$$

In particular, using these inequalities with $q \leq 1$, we see that the "norms" \|\|$p\left\|^{\frac{1}{d}}\right\|_{L^{q}(K)}$ for $q \leq 1$ and $\left[\sup _{\alpha>0} \alpha^{-\frac{1}{d}}|\{x \in K:\|p(x)\| \leq \alpha\}|\right]^{-1}=$ \|\|$p\left\|^{-1 / d}\right\|_{L^{1, \infty}(K)}^{-1}$ are uniformly equivalent. Therefore for any $q \geq r$, we have

$$
\left(\int_{K}\|p(x)\|^{\frac{q}{d}} d x\right)^{\frac{1}{q}} \leq C n(n B(n, q+1))^{\frac{1}{q}}\left(\int_{K}\|p(x)\|^{\frac{r}{d}} d x\right)^{\frac{1}{r}} .
$$

3. It is easy to see that the conclusion of Theorem 2 has the following equivalent formulation for general finite-volume convex bodies $K$ :

$$
\left(\frac{1}{|K|} \int_{K}\|p\|^{\frac{q}{d}}\right)^{\frac{1}{q}} \leq C n(n B(n, q+1))^{\frac{1}{q}} \frac{|K|}{|E|}\|\| p\left\|^{\frac{1}{d}}\right\|_{L^{\infty}(E)}
$$

uniformly over all closed subsets $E$ of $K$ (with the same constant $C$ ). Somewhat surprisingly, one can replace the $L^{\infty}$ norm on the right side with the smaller $L^{r}$ norm, $\left(\frac{1}{|E|} \int_{E}\|p\|^{\frac{r}{d}}\right)^{\frac{1}{r}}$, incurring only an extra factor of 2 in the estimate. This was observed in [BG] for the case $q=\infty$ and follows by considering the non-decreasing rearrangement of $\|p\|$ over $E, p_{*}(\tau)$ (i.e., $p_{*}$ is the inverse of the measure of the sublevel sets of $\|p\|$ restricted to $E$ ). The estimate in Theorem 2 implies a lower bound for $p_{*}$, namely

$$
\left(\frac{1}{|K|} \int_{K}\|p\|^{\frac{q}{d}}\right)^{\frac{1}{q}} \frac{\tau}{|K|} \leq C n(n B(n, q+1))^{\frac{1}{q}}\left[p_{*}(\tau)\right]^{\frac{1}{d}}
$$

for $0 \leq \tau \leq|E|$. Raising this to the $r^{\prime}$ 'th power, integrating in $\tau$ and then taking the $r$ 'th root gives the desired bound.

4. The convexity of the set $K$ is crucial in obtaining the form of the constant in Theorem 1. If instead one asks for the form of the constant $B$ in the inequality

$$
\left(\int_{F}\|p\|^{\frac{q}{d}}\right)^{\frac{1}{q}} \leq B\left(\int_{F}\|p\|^{\frac{r}{d}}\right)^{\frac{1}{r}}
$$


where $F$ is now an arbitrary (unit-volume) compact set in $\mathbb{R}^{n}$ and $0 \leq r \leq q \leq$ $\infty$, one may see that not only must $B$ contain a factor of $|c v x F|^{1-\frac{r}{q}}$ (where $\operatorname{cvx} F$ denotes the convex hull of $\mathrm{F}$ ) but also a factor $n^{1-\frac{r}{q}}$. To see this, consider the example $p(x)=x_{1}^{d}$ as before and $F=\left\{\left(x_{1}, x^{\prime}\right) \in \mathbb{R}^{n}: x_{1} \in(0,1 / n) \cup\right.$ $\left.(1-\epsilon, 1),\left|x^{\prime}\right| \leq 1-x_{1}\right\}$ for suitable $\epsilon$ much smaller than $1 / n$. The proof of the resulting inequality

$$
\left(\int_{F}\|p\|^{\frac{q}{d}}\right)^{\frac{1}{q}} \leq C n^{1-\frac{r}{q}}|c v x F|^{1-\frac{r}{q}}\left(\int_{F}\|p\|^{\frac{r}{d}}\right)^{\frac{1}{r}}
$$

is due to Brudnyi and Ganzburg [BG] (at least in the case $q=\infty$ ). To see this, we first observe

$$
\begin{gathered}
\int_{F}\|p(x)\|^{\frac{q}{d}} \leq \int_{F}\|p(x)\|^{\frac{r}{d}}\|\| p\left\|^{\frac{1}{d}}\right\|_{L^{\infty}(F)}^{q-r} \leq \int_{F}\|p(x)\|\left\|^{\frac{r}{d}}\right\|\|p\|^{\frac{1}{d}} \|_{L^{\infty}(c v x F)}^{q-r} \\
\leq \int_{F}\|p(x)\|^{\frac{r}{d}}\left\{\frac{1}{(n B(n, r+1))^{\frac{1}{r}}}\|\| p\left\|^{\frac{1}{d}}\right\|_{L^{r}(\operatorname{cvx} F)}\right\}^{q-r}
\end{gathered}
$$

where the last inequality follows from the case $q=\infty$ and general $r$ of Theorem 1. Next, using the following equivalent formulation of Theorem 2 (which we derived in Remark 3 above)

$$
\left(\frac{1}{|K|} \int_{K}\|p\|^{\frac{q}{d}}\right)^{\frac{1}{q}} \leq C n(n B(n, q+1))^{\frac{1}{q}} \inf _{E \subset K} \frac{|K|}{|E|}\left(\frac{1}{|E|} \int_{E}\|p\|^{\frac{r}{d}}\right)^{\frac{1}{r}}
$$

when $q=r, E=F$ and $K=c v x F$, we obtain the result. Interestingly, (3) can be thought of as a way to formulate the analogue of Lemma 1 in the higherdimensional context, and it is natural to enquire as to whether the constant $n(n B(n, q+1))^{\frac{1}{q}}$ can be improved upon if we restrict $E$ to range over convex subsets of $K$. This however is not the case. To see this, take $K=\left\{\left(x_{1}, x^{\prime}\right) \in\right.$ $\left.\mathbb{R}^{n}: 0<x_{1}<n-1,\left|x^{\prime}\right|<1-\frac{1}{n-1} x_{1}\right\}, E=\left\{\left(x_{1}, x^{\prime}\right) \in \mathbb{R}^{n}: 0<x_{1}<1,\left|x^{\prime}\right|<\right.$ $\left.1-\frac{1}{n-1} x_{1}\right\}$, and $p(x)=x_{1}^{d}$. (Of course, $X=\mathbb{R}$ here).

5. If $p: \mathbb{R}^{n} \rightarrow \mathbb{C}$ is a polynomial of degree at most $d$, it is well known that $\omega=|p|$ is an $A_{q}$ weight when $q>d+1$ with $A_{q}$ bounds independent of the coefficients of $p$; see [RS]. Theorem 2 , when $q=d$, can be viewed as a sharp endpoint result of this nature. Recall that a weight $\omega$ is in $A_{q}$ if

$$
\frac{1}{|B|} \int_{B} \omega(x) d x \cdot\left[\frac{1}{|B|} \int_{B} \omega(x)^{-q^{\prime} / q} d x\right]^{q / q^{\prime}} \leq A<\infty
$$

for all balls $B$ in $\mathbb{R}^{n}$. The smallest constant $A$ for which the above holds is called the $A_{q}$ bound, $A_{q}(\omega)$, for $\omega$. Using Theorem 2 with $q=d$, we see that there is an absolute constant $C$ such that if $p: \mathbb{R}^{n} \rightarrow X$ is a polynomial of degree at most $d$ with values in a Banach space $X$ and $q>d+1$,

$$
A_{q}(\|p\|) \leq(C d)^{d}\left[\frac{q-1}{q-(d+1)}\right]^{q-1} .
$$


We remark that this estimate remains valid when we allow the $A_{q}$ bound to also vary over all convex bodies $K$ in $\mathbb{R}^{n}$, not just Euclidean balls $B$. See also [NSV].

6. The theorem of Kannan, Lovász and Simonovits which we used relies heavily on the non-negativity of the functions involved. However there are phenomena, closely related to sublevel set problems for polynomials, which are highly oscillatory in nature; most notably estimates for oscillatory integrals. For example, it follows from Theorem 7.2 of [CCW] that if $Q=[0,1]^{n}, p: Q \rightarrow \mathbb{R}$ is a polynomial of degree at most $d$ so that $\int_{Q} p=0$ and $\|p\|_{L^{\infty}(Q)}=1$, then for $\lambda$ large and real,

$$
\left|\int_{Q} \mathrm{e}^{i \lambda p(x)} d x\right| \leq \frac{C_{d, n}}{|\lambda|^{\frac{1}{d}}}
$$

Can we expect improvement to this along the lines enjoyed by sublevel sets? In particular if $\int_{Q} p=0$ and $\|p\|_{L^{1}(Q)}=1$, can we take $C_{d, n}$ in (4) to be $C \min (d, n)$ ? On average the answer is yes, because a direct consequence Theorem 2 is that for $\|p\|_{L^{1}(K)}=1, K$ a convex body of volume $1, \phi \in \mathcal{S}(\mathbb{R})$ with $0 \leq \hat{\phi} \leq \chi_{[-1,1]}$

$$
\left|\frac{1}{\mu} \int\left\{\int_{K} \mathrm{e}^{i \lambda p(x)} d x\right\} \phi(\lambda / \mu) d \lambda\right| \leq C \min (d, n) \mu^{-\frac{1}{d}}
$$

with $C$ absolute. To see this, note that the left side is equal to $\int_{K} \hat{\phi}(\mu p(x)) d x$ which is in turn equivalent to $\left|\left\{x \in K:|p(x)| \leq \mu^{-1}\right\}\right|$. (This well-known argument also demonstrates the fact that oscillatory integral estimates imply sublevel set estimates).

\section{Acknowledgements}

We would like to thank A. Giannopoulos and A. Volberg for bringing these problems to our attention. In fact, the present authors' interest in these problems principally arose upon receiving in July 2000 a preprint "Dimensionless $A_{p}$ bounds and the distribution of polynomials of many real variables" by M. Sodin and A. Volberg, which later evolved into [NSV]. In this preprint the problem of transference of $A_{p}$ estimates through dimensions was considered, and a version of our current Theorem 2 in the case $q=0$, but with extra logarithmic terms in $\alpha$ was given. We record our debt of thanks to the authors of [NSV] for generously sharing their work with us and also for drawing to our attention several references. Finally we thank the referee for various helpful comments.

\section{References}

[Bobk] S. G. Bobkov, Remarks on the growth of $L^{p}$ - norms of polynomials, Lecture Notes in Math. 1745 (2000), Springer, 27-35.

[Bour] J. Bourgain, On the distribution of polynomials on high dimensional convex sets, Lecture Notes in Math. 1469 (1991), Springer, 127-137. 
[BG] Yu. A. Brudnyi and M. I. Ganzburg, A certain extremal problem for polynomials in $n$ variables, (Russian) Izv. Akad. Nauk SSSR Ser. Mat. 37 (1973), 344-355, English translation: Math. USSR-Izv. 7 (1973), 345-356.

[CCW] A. Carbery, M. Christ and J. Wright, Multidimensional van der Corput and sublevel estimates, J. Amer. Math. Soc 12 (1999), 981-1015.

[C] H. Cartan, Sur les systèmes de fonctions holomorphes à variétés linéaires lacunaires et leurs applications, Ann. Sci. École Norm. Sup. 45 (1928), 225-346.

[DR] R. M. Dudley and B. Randol, Implications of pointwise bounds on polynomials, Duke Math. J. 29 (1962), 455-458.

[GM] M. Gromov and V. D. Milman, Brunn theorem and a concentration of volume for convex bodies, Israel seminar on geometrical aspects of functional analysis (1983/84), Tel Aviv University, Tel Aviv, 1984.

[H] W. H. Hayman, Subharmonic Functions, vol. 2, London Mathematical Society Monographs 20, Academic Press, London, 1989.

[KLS] R. Kannan, L. Lovász and M. Simonovits, Isoperimetric problems for convex bodies and a localization lemma, Discrete Comput. Geom. 13 (1995), 541-559.

[NSV] F. Nazarov, M. Sodin, and A. Volberg, The geometric KLS lemma, dimension-free estimates for the distribution of values of polynomials and distribution of zeroes of random analytic functions, preprint.

[RS] F. Ricci and E. M. Stein, Harmonic analysis on nilpotent groups and singular integrals I: Oscillatory integrals, J. Funct. Anal. 73 (1987), 179-194.

Department of Mathematics and Statistics, University of Edinburgh, JCMB, King's Buildings, Mayfield Road, Edinburgh EH9 3JZ, Scotland.

E-mail address: carbery@maths.ed.ac.uk

E-mail address: wright@maths.ed.ac.uk 